Research Article

\title{
Ginsenoside Re Improves Inflammation and Fibrosis in Hepatic Tissue by Upregulating PPAR $y$ Expression and Inhibiting Oxidative Stress in $\mathbf{d b} / \mathbf{d b}$ Mice
}

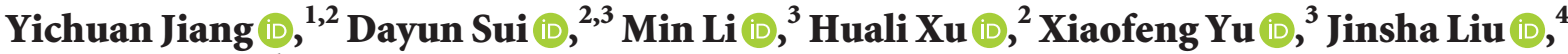 \\ and Qian $\mathbf{Y u} \mathbb{D}^{1}$ \\ ${ }^{1}$ Department of Pharmacy, China-Japan Union Hospital, Jilin University, Changchun 130033, China \\ ${ }^{2}$ Department of Pharmacology, School of Pharmaceutical Sciences, Jilin University, Changchun 130021, China \\ ${ }^{3}$ Pharmacological Experiment Center, School of Pharmaceutical Sciences, Jilin University, Changchun 130021, China \\ ${ }^{4}$ Department of Cardiology, China-Japan Union Hospital, Jilin University, Changchun 130033, China
}

Correspondence should be addressed to Qian Yu; yuqian@jlu.edu.cn

Received 15 April 2021; Accepted 25 September 2021; Published 8 October 2021

Academic Editor: Junqing Yang

Copyright $\odot 2021$ Yichuan Jiang et al. This is an open access article distributed under the Creative Commons Attribution License, which permits unrestricted use, distribution, and reproduction in any medium, provided the original work is properly cited.

Ginsenoside Re (Re) is the main component of "Zhenyuan Capsule" (ZYC), which was wildly used in clinic in China for adjunctive treatment of coronary heart disease (CHD) and type II diabetes (T2DM). Nonalcoholic fatty liver disease (NAFLD) is one of the most important complications of T2DM, as well as an important risk factor of CHD. The aim of the present study was to investigate the effects of Re on NAFLD in $\mathrm{db} / \mathrm{db}$ mice, one of the most recognized gene deficient animal models on T2DM. Sixteen $\mathrm{db} / \mathrm{db}$ mice and sixteen wild-type mice were divided into four groups and orally administered Re or placebo in equal volume. According to the results, Re showed no obvious effect on blood glucose, lipids, or body weight of $\mathrm{db} / \mathrm{db}$ mice. Histology pictures of hepatic tissue showed that Re did not improve steatosis, too. However, some evidence suggested that hepatic injury in $\mathrm{db} / \mathrm{db}$ mice was attenuated by Re administering. Collagen deposition and aminotransferase elevation were significantly downregulated in the DB + Re group compared to those in the DB Group. The mechanisms of the protect effects of Re represented in $\mathrm{db} / \mathrm{db}$ mice with NAFLD might be inhibiting oxidative stress and the reupregulation of peroxisome proliferator-activated receptor $\gamma$ (ppar $\gamma$ ) expression. The results of this study indicated that ZYC might be able to help T2DM patients with NAFLD to control the progress of NAFLD as an alternation of thiazolidinediones, synthetic agonists of $\operatorname{PPAR} \gamma$, whose side effects and adverse events should not be ignored.

\section{Introduction}

Ginsenoside $\operatorname{Re}(\mathrm{Re})$ is one of the protopanaxatriol-type saponins (Figure 1), which are abundant in Araliaceae plants such as Panax ginseng C.A. Meyer (ginseng) and Panax quinquefolium L. (American ginseng). Especially, Re is the essential component of total saponins extracted from the fruit of ginseng, which had been developed as a drug named "Zhenyuan Capsule" (ZYC). ZYC was wildly used in clinic in China for adjunctive treatment of coronary heart disease (CHD) and type II diabetes (T2DM), especially CHD with abnormal glucose and lipid metabolism [1]. Current evidence showed that ZYC combined with routine treatment could improve the cardiac function and quality of life of patients with chronic heart failure, and with high safety [2]. The content of Re is also the major technical standard in the quality control of ZYC.

Our group is currently undertaking a long-term study assessing the effects of various ginsenosides, including Re, in various animal models of chronic disease, such as hypertension [3, 4], CHD [5-7], and T2DM. Especially, we had demonstrated that Re improves acute myocardial ischemiamediated myocardial injury, myocardial fibrosis, and heart failure. Regarding the effects of Re on CHD, the mechanisms might be associated with inhibiting oxidative stress and transforming growth factor- $\beta$ - (TGF- $\beta$-) mediated fibrosis in the myocardium in rats $[6,7]$. As $\mathrm{Re}$ is the essential component of ZYC, which has been widely used as an 


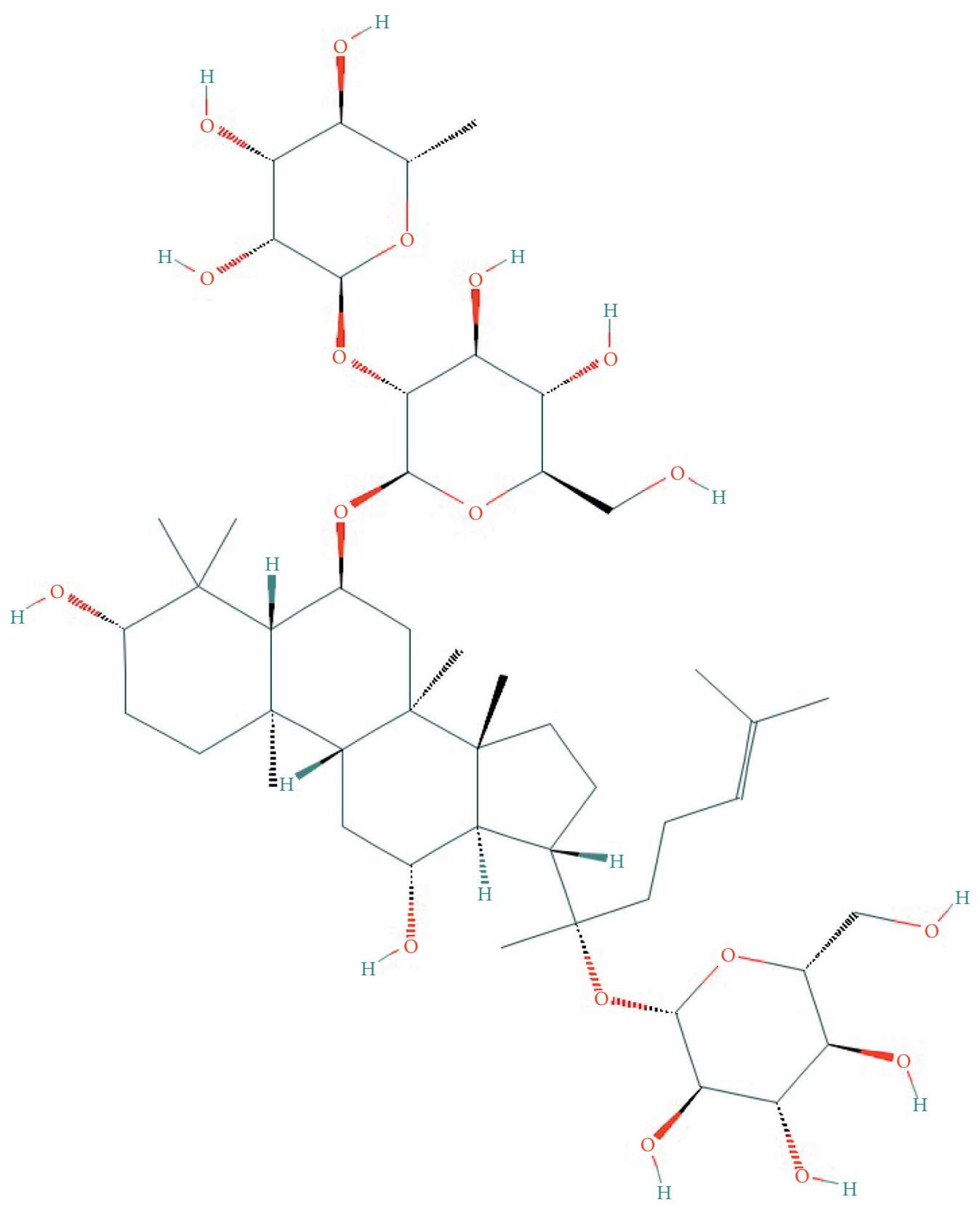

Figure 1: Chemical structure of Re, downloaded from PubChem (https://www.pubchem.ncbi.nlm.nih.gov), CID 441921.

adjunctive treatment of CHD and T2DM, in this study, we determined the protective effects of Re on nonalcoholic fatty liver disease (NAFLD), which has a complex relationship with CHD and T2DM.

Studies have shown that, up to $70 \%$ of patients with diabetes might present NAFLD, which often results in abnormal liver function [8-10]. Studies have also demonstrated that NAFLD is an important risk factor for higher prevalence of cardiovascular diseases, for example, CHD $[9,11]$. According to the "multihit hypothesis" of NAFLD [12-16], insulin resistance within the liver is implicated in the pathogenesis of NAFLD. Upregulation of the peroxisome proliferator-activated receptor gamma (PPAR $\gamma$ ) pathway could attenuate inflammation and fibrosis in hepatic tissue as well as insulin resistance, and these had been confirmed by preclinic animal research in vivo or clinic research $[8,17,18]$. Thiazolidinediones (TZDs), synthetic agonists of PPAR $\gamma$, are widely used in clinic for improving hepatic injury in NAFLD patients [11, 19-21].

PPAR $\gamma$ agonists have been demonstrated to improve hepatic inflammation and fibrosis in T2DM animal models with NAFLD $[22,23]$. In this study, we were going to observe the hepatic protective effects of $\mathrm{Re}$ and determined the expression of PPAR $\gamma$ in hepatic tissue in $\mathrm{db} / \mathrm{db}$ mice (BKSLeprem2Cd479/Gpt), one of the most recognized gene deficient animal models on T2DM with the complication of NAFLD. The leptin receptor is deficient in $\mathrm{db} / \mathrm{db}$ mice, which results in obesity, hyperglycemia, hyperlipidemia, and hepatic steatosis $[24,25]$. Wild-type (WT) mice with the same genetic background (C57BLKS/JGpt) were used in the 
two control groups. The effects of Re on oxidative stress, inflammation, and fibrosis in hepatic tissue were also determined, as we had demonstrated that Re improves myocardial injury in $\mathrm{CHD}$ by inhibiting oxidative stress, inflammation, and fibrosis [6, 7].

\section{Materials and Methods}

2.1. Chemicals and Reagents. Re (95\% purity) was obtained from Dr. Yanping Chen at the Department of Natural Medicinal Chemistry, School of Chemistry, Jilin University. It was dissolved in $0.5 \%$ sodium carboxymethyl cellulose solution $(0.5 \% \mathrm{CMC}-\mathrm{Na})$ for use. All of the other chemicals and reagents were of analytical grade.

2.2. Animals. $16 \mathrm{db} / \mathrm{db}$ (abbreviated as $\mathrm{DB}$ in group names) mice and 16 WT mice were purchased from Nanjing Biomedical Research Institute of Nanjing University (Nanjing, China). All of the mice were male and 12 13-weeks old. The experimental animal house was of specific pathogen free (SPF) grade. The constant temperature in the house was $22-24^{\circ} \mathrm{C}$, and the relative humidity was $45-55 \%$. All the mice had free access to water and food. The light/dark cycle was 12 hours/12 hours.

2.3. Experimental Protocols. All the mice were divided into 4 groups: (I) group WT, 8 WT mice; (II) group WT + Re, 8 WT mice; (III) group DB, $8 \mathrm{db} / \mathrm{db}$ mice; and (IV) group $\mathrm{DB}+\mathrm{Re}$, $8 \mathrm{db} / \mathrm{db}$ mice. Mice in the II and IV groups were administered Re at the doze of $30 \mathrm{mg} \mathrm{kg}^{-1} \mathrm{~d}^{-1}$ orally, while mice in the I and III groups were administered 0.5\% CMC-Na as placebo orally. In our previous studies $[6,7]$, rats were orally administered $20 \mathrm{mg} \mathrm{kg}^{-1} \mathrm{~d}^{-1}$ Re. Therefore, the biological equivalent dose of $\mathrm{Re}$ in mice is $30 \mathrm{mg} \mathrm{kg}^{-1} \mathrm{~d}^{-1}$. Administration of placebo or Re was carried on for 8 weeks. During these 8 weeks, blood glucose and body weight of mice were measured weekly.

After the 8-week treatment, all the mice in the 4 groups were sacrificed following the blood sample collection. Then, the tissue samples of the livers were obtained. The blood samples of mice were prepared into serum by centrifugation $\left(1000 \mathrm{~g}, 4^{\circ} \mathrm{C}\right.$ for 15 minutes) and kept at $-80^{\circ} \mathrm{C}$. The hepatic tissue samples were fixed in $4 \%$ formaldehyde or snapfrozen with liquid nitrogen. The samples fixed in formaldehyde were embedded in paraffin, and the snap-frozen samples were kept at $-80^{\circ} \mathrm{C}$.

2.4. Blood Glucose Measurement. Lateral tail vein blood glucose was measured using the Fasting Blood Glucose Test Kit (GlucoLab, Infopia Co., Ltd., Kyunggi, Korea) following the manufacturer's protocol weekly during the 8-week treatment.

2.5. Serum Biochemical Assays. Biochemical assay kits were purchased form Nanjing Jiancheng Bioengineering Institute (Nanjing, China): ALT (alanine aminotransferase, C009-21), AST (aspartate aminotransferase, C010-2-1), TG (triglyceride, A110-1-1), TC (total cholesterol, A111-1-1), HDL (high-density lipoprotein cholesterol, A112-1-1), and LDL (low-density lipoprotein cholesterol, A113-1-1). The activities of ALT and AST and levels of TG, TC, HDL, and LDL in serum were assayed following the manufacturer's protocols.

2.6. Hepatic Tissue Biochemical Assays. Hepatic tissue samples kept at $-80^{\circ} \mathrm{C}$ were homogenized with ice-cold normal saline (100 mg: $900 \mu \mathrm{L}$ ), and the supernatant samples were prepared for biochemical assays by centrifuging (1000 g, $4^{\circ} \mathrm{C}$ for 15 minutes). Biochemical assay kits were purchased form Nanjing Jiancheng Bioengineering Institute (Nanjing, China): MDA (malondialdehyde, A003-1-1), SOD (superoxide dismutase, A001-3-1), CAT (catalase, A007-11), and GSH-Px (glutathione peroxidase, A005-1-2). The levels of MDA and activities of SOD, CAT, and GSH-Px in hepatic tissue were assayed following the manufacturer's protocols.

2.7. Histopathological Assessment. Hepatic tissue samples embedded in paraffin were cut into $4 \mu \mathrm{m}$-thick sections for hematoxylin and eosin (H\&E) or Masson trichrome (Masson) staining. The Masson kit (BSBA-4079A, ZSGB$\mathrm{BIO}$, Beijing, China) was used following the manufacturer's protocols for Masson staining.

Hepatic tissue samples kept at $-80^{\circ} \mathrm{C}$ were cut into $6 \mu \mathrm{m}$ thick cryostat sections for Oil-Red-O (ORO) staining of lipid droplets. The ORO kit (BSBA-4081, ZSGB-BIO, Beijing, China) was used following the manufacturer's protocols for ORO staining.

All the stained sections were examined using a Nikon E100 light microscope (Nikon Corporation, Tokyo, Japan); then, histopathology pictures were captured.

2.8. Immunohistochemistry. Staining of immunohistochemistry (IHC) was performed using the two-step rabbit IHC kit (PV-9001, ZSGB-BIO, Beijing, China) and DAB kit (ZLI-9017, ZSGB-BIO, Beijing, China) following the manufacturer's protocols. Primary antibodies used in staining were anti-PPAR- $\gamma$ (bs-4590R, Bioss Inc., Beijing, China) and anti-TGF- $\beta 1$ (bs-0103R, Bioss Inc., Beijing, China). All the stained sections were examined using the microscope mentioned above; then, histopathology pictures were captured and further analyzed using Image Pro Plus 6.0 (Media Cybernetics, Inc., Rockville, MD, USA).

2.9. RNA Purification and RT-qPCR. Total RNA was isolated from frozen hepatic tissue samples using TRIzol reagent (Thermo Fisher Scientific, Inc., MA, USA) following the manufacturer's protocol. Total RNA was reverse-transcribed, and qPCR was conducted using the TransScript Green Two-Step qRT-PCR SuperMix (TransGen Biotech Co., Ltd., Beijing, China) on a Stratagene Mx3000P RealTime PCR System (Agilent Technologies, Inc., CA, USA). The relative fold changes in the mRNA levels of the target genes, including tumor necrosis factor- $\alpha$ (TNF- $\alpha$ ), 
interleukin-6 (IL-6), procollagen I (Col-I), and procollagen III (Col-III), were determined using the $2^{-\Delta \Delta C t}$ method [26]. $\beta$-Actin was used as a housekeeping gene. All primers are listed in Table 1.

2.10. Statistical Analysis. SPSS 16.0 (IBM Corporation, NY, USA) was employed for all statistical analyses. Data are mean \pm standard deviation (SD). One-way analysis of variance (ANOVA) with Tukey's post hoc test was employed for group comparisons, with $P<0.05$ indicating statistical significance.

\section{Results}

3.1. Re Has No Significant Effect on Body Weight, Blood Glucose, and Lipids. The mice in the WT group and WT + Re group did not present a significant difference regarding body weight, blood glucose, and four kinds of lipids (TG, TC, HDL, and LDL). The situation was the same between the DB group and $\mathrm{DB}+\mathrm{Re}$ group. But, the levels of body weight, blood glucose, and lipids are all significantly higher in $\mathrm{db} / \mathrm{db}$ mice than in WT mice, no matter being administered of Re or not (Figure 2).

3.2. Re Reduces Activities of Aminotransferases in Serum. In the DB group, activities of ALT and AST in serum were significantly higher than the activities in the WT Group. The disorder of glucose and lipid metabolism resulted in hepatocytes injury and aminotransferase leakage in $\mathrm{db} / \mathrm{db}$ mice. Comparing to the $\mathrm{DB}$ group, Re administration markedly reduced the activities in the $\mathrm{DB}+\mathrm{Re}$ group, while the activities in the $\mathrm{WT}+\mathrm{Re}$ group were not significantly downregulated comparing to those in the WT group (Figure 3). These results indicated that $\mathrm{Re}$ administration attenuated hepatocytes injury and aminotransferase leakage in $\mathrm{db} / \mathrm{db}$ mice.

3.3. Re Inhibits Oxidative Stress in Hepatic Tissue. Compared to the WT group, the activities of SOD, CAT, and GSH-Px in hepatic tissue in the DB group were significantly downregulated, while the MDA levels in the DB group were significantly upregulated. These changes indicated that oxidative stress existed in hepatic tissue of $\mathrm{db} / \mathrm{db}$ mice. Oxidative stress was inhibited by $\mathrm{Re}$ administration, as the activities of SOD, CAT, and GSH-Px were significantly upregulated and MDA levels were significantly downregulated in the $\mathrm{DB}+\mathrm{Re}$ group, compared to those in the $\mathrm{DB}$ Group. There was no significant difference in the results of these biochemical tests between the WT group and WT $+\mathrm{Re}$ group (Figure 4).

3.4. Re Does Not Reverse Simple Steatosis but Inhibits Fibrosis in Hepatic Tissue. According to the results of H\&E, Masson, and ORO staining examination, the hepatic tissue specimens in both the $\mathrm{DB}$ group and $\mathrm{DB}+\mathrm{Re}$ group represented obvious steatosis. The morphology of hepatic lobules was also disordered in both of these two groups. All of these
TABle 1: Primer sequence for RT-qPCR.

\begin{tabular}{lcc}
\hline Gene & & Sequence (5'-3') \\
\hline \multirow{2}{*}{$\beta$-Actin } & $\begin{array}{c}\text { Forward } \\
\text { Reverse }\end{array}$ & $\begin{array}{c}\text { GGCTGTATTCCCCTCCATCG } \\
\text { CCAGTTGGTAACAATGCCATGT }\end{array}$ \\
\hline \multirow{2}{*}{ TNF- $\alpha$} & $\begin{array}{c}\text { Forward } \\
\text { Reverse }\end{array}$ & $\begin{array}{c}\text { GTCGTAGCAAACCACCAAGT } \\
\text { TGTGGGTGAGGAGCACGTAG }\end{array}$ \\
\hline \multirow{2}{*}{ IL-6 } & $\begin{array}{l}\text { Forward } \\
\text { Reverse }\end{array}$ & $\begin{array}{c}\text { GTCCTTCAGAGAGATACAGAAACT } \\
\text { AGCTTATCTGTTAGGAGAGCATTG }\end{array}$ \\
\hline \multirow{2}{*}{ Col-I } & $\begin{array}{c}\text { Forward } \\
\text { Reverse }\end{array}$ & CTTCACCTACAGCACCCTTGTG \\
\hline \multirow{2}{*}{ Col-III } & $\begin{array}{c}\text { Forward } \\
\text { Reverse }\end{array}$ & $\begin{array}{c}\text { TGTCCTTTGCTTGCCCCAAGTTC } \\
\text { AATGGGATCTCTGGGTTGGG }\end{array}$ \\
\hline
\end{tabular}

pathological changes did not exist in the WT group or WT + Re group. However, the Masson staining examination showed that collagen was deposited in hepatic tissue in the $\mathrm{DB}$ group, while it was markedly improved in the DB + Re group (Figure 5). These results demonstrated that Re has an antifibrosis effect on the liver of $\mathrm{db} / \mathrm{db}$ mice, but Re does not reverse simple steatosis.

3.5. Re Upregulates PPAR $\gamma$ and Downregulates TGF- $\beta 1$ Expression in Hepatic Tissue. According to the IHC examination, the expression levels of PPAR $\gamma$ were significantly higher in hepatic tissue specimens from the DB group than those from the WT Group. They were compensated upregulated because of glucose and lipid metabolism. Interestingly, the levels were even further upregulated in the DB + Re group by $\mathrm{Re}$ administration, improving insulin resistance, inflammation, and fibrosis.

The situation of TGF- $\beta 1$ was different. As an important factor on inflammation and fibrosis, TGF- $\beta 1$ was over expressed in hepatic tissue in mice from the DB group, compared to that from the WT group. Also, the expression of TGF- $\beta 1$ was significantly downregulated by Re administration in the $\mathrm{DB}+\mathrm{Re}$ group, compared to that in the $\mathrm{DB}$ group.

The expression levels of PPAR $\gamma$ or TGF- $\beta 1$ in hepatic tissue specimens from the $\mathrm{WT}+\mathrm{Re}$ group did not represent a significant difference compared to those from the WT group (Figure 6).

3.6. Re Reduces Levels of Inflammatory Cytokines and Procollagens in Hepatic Tissue. RT-qPCR was used to measure the relative levels of mRNA in hepatic tissue (Figure 7), including two inflammatory cytokines (TNF- $\alpha$ and IL-6) and two procollagens (Col-I and Col-III). The levels of TNF$\alpha$, IL-6, Col-I, and Col-III were all significantly higher in the DB group, compared to those in the WT group, while those levels from the WT + Re group did not represent a significant difference compared to those from the WT group. The levels of two inflammatory cytokines were significantly downregulated in the $\mathrm{DB}+\mathrm{Re}$ group compared to those in the $\mathrm{DB}$ group, but were still significantly higher compared to those in the WT Group. The levels of two procollagens were also significantly downregulated by $\operatorname{Re}$ administration in the 


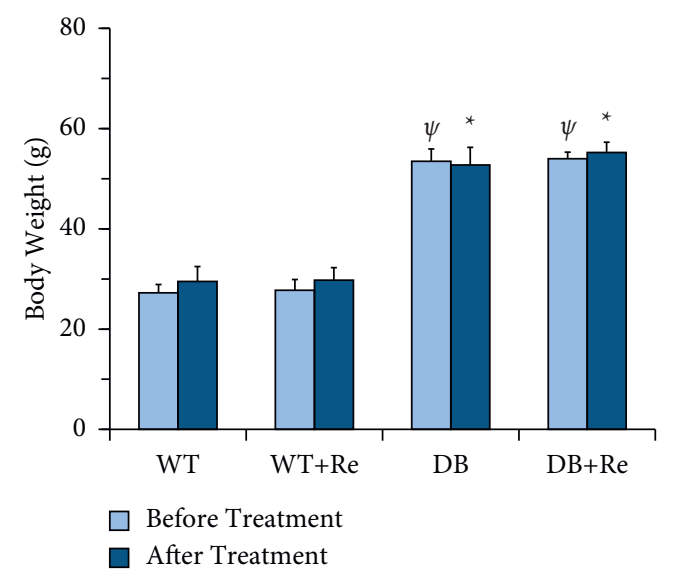

(a)

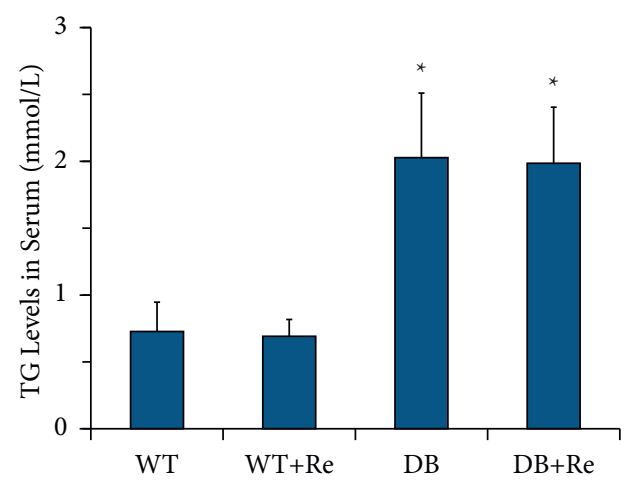

(c)

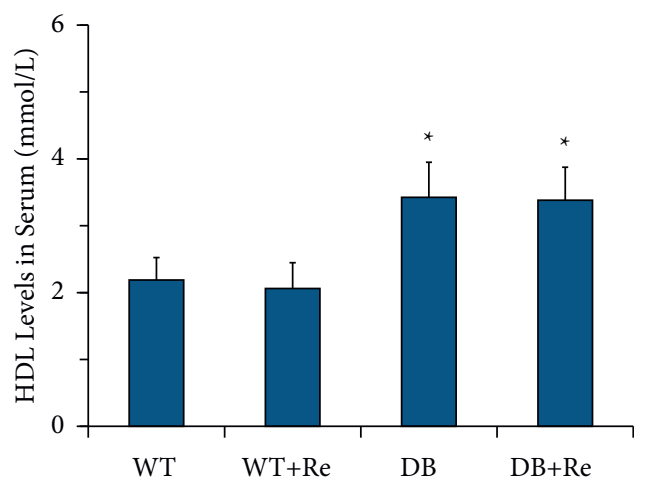

(e)

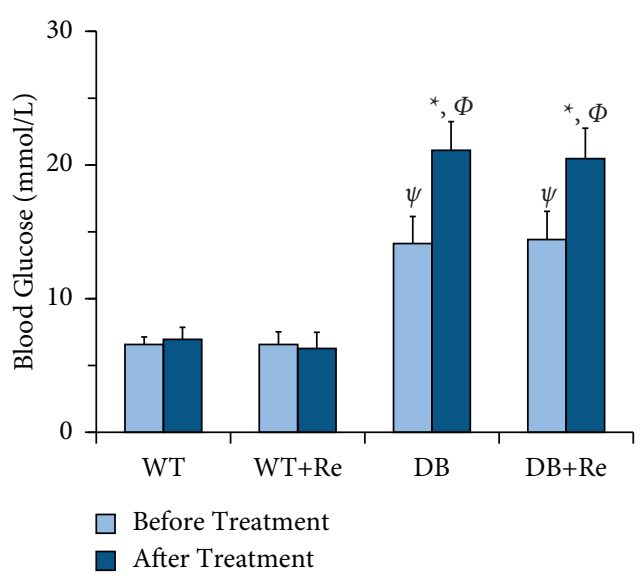

(b)

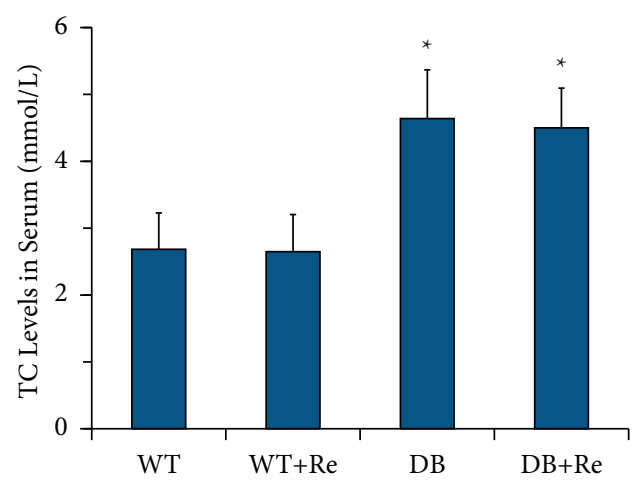

(d)

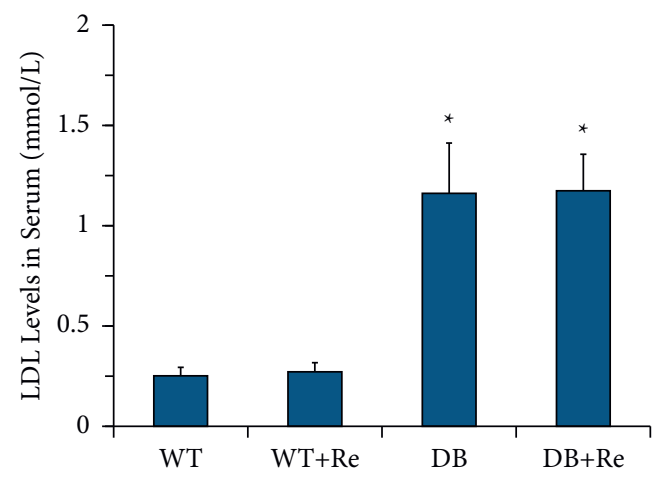

(f)

Figure 2: Re has no significant effect on body weight, blood glucose, and lipids. (a) Body weight of mice prior to and following eight weeks of treatment; (b) blood glucose of mice prior to and following eight weeks of treatment; (c) TG levels in serum in mice after treatment; (d) TC levels in serum in mice after treatment; (e) HDL levels in serum in mice after treatment; and (f) LDL levels in serum in mice after treatment. Data are mean $\pm \mathrm{SD}$, and $n=8$. One-way ANOVA with Tukey's post hoc test was employed for group comparisons, with $P<0.05$ indicating statistical significance. $\Psi, P<0.05$ compared to the WT group before treatment; ${ }^{*} P<0.05$ compared to the WT group after treatment; $\Phi$, $P<0.05$ compared to the DB group before treatment; and $\Omega, P<0.05$ compared to the $\mathrm{DB}+\mathrm{Re}$ group before treatment.

$\mathrm{DB}+$ Re group, and they did not represent significant difference compared to those in the WT Group.

\section{Discussion}

As it is mentioned above, NAFLD is one of the most common causes of abnormal liver function among adults. It is also one of the most common and important complications of T2DM, as well as an important risk factor of CHD. NAFLD usually develops from simple steatosis to nonalcoholic steatohepatitis (NASH), which can progress to end-stage liver disease [9]. Thus, it is important to improve early hepatic injury and prevent simple steatosis from developing into NASH or hepatic fibrosis.

ZYC, whose essential component is Re, has been widely used for T2DM and CHD treatment in clinic in China. 


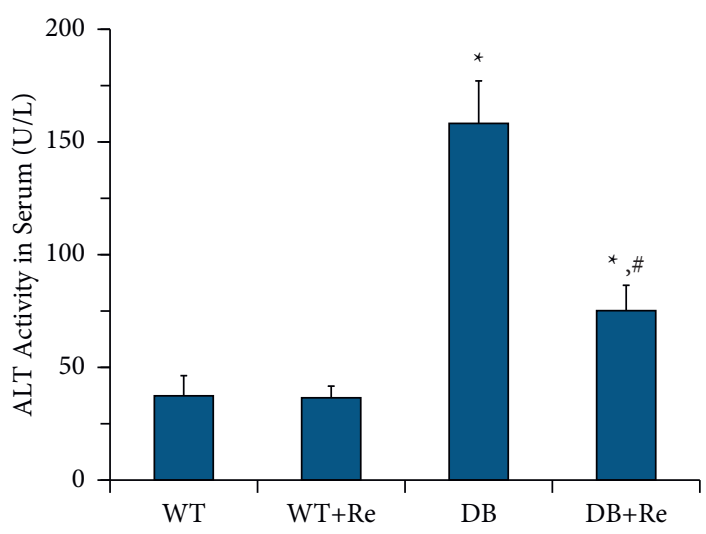

(a)

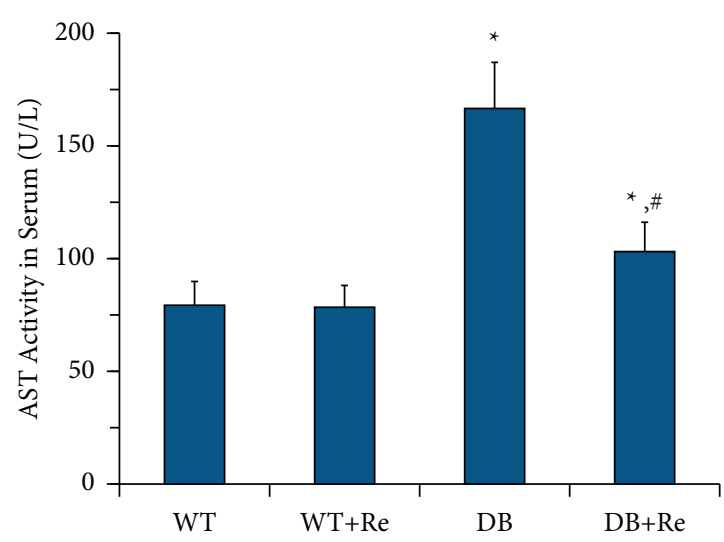

(b)

Figure 3: Re reduces activities of aminotransferases in serum. (a) Activities of ALT in serum; (b) activities of AST in serum. Data are mean $\pm \mathrm{SD}$, and $n=8$. One-way ANOVA with Tukey's post hoc test was employed for group comparisons, with $P<0.05$ indicating statistical significance. ${ }^{*} P<0.05$ compared to the WT group; ${ }^{\#} P<0.05$ compared to the DB group.

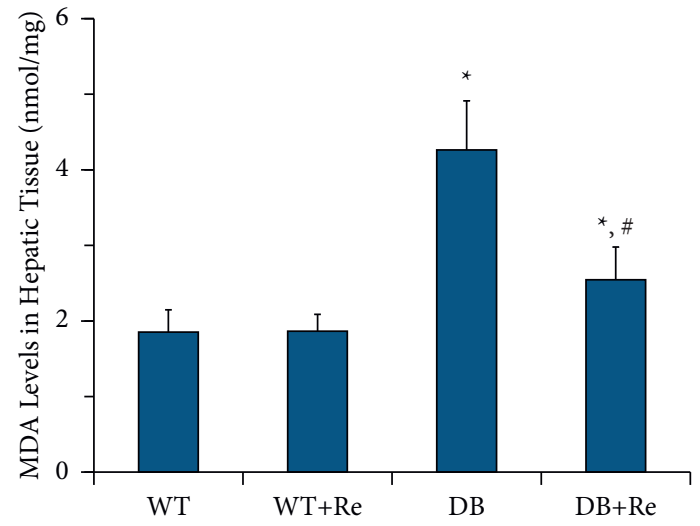

(a)

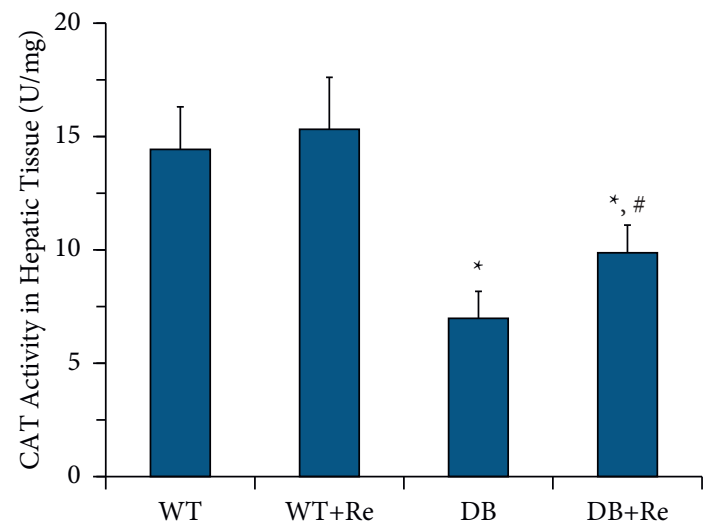

(c)

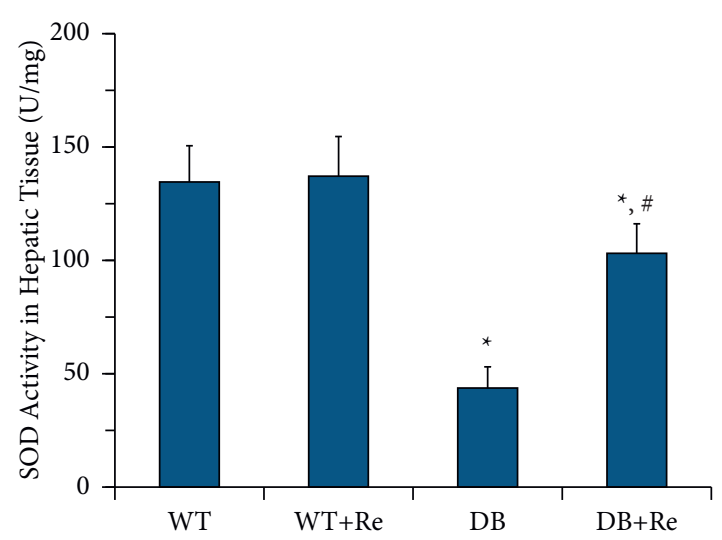

(b)

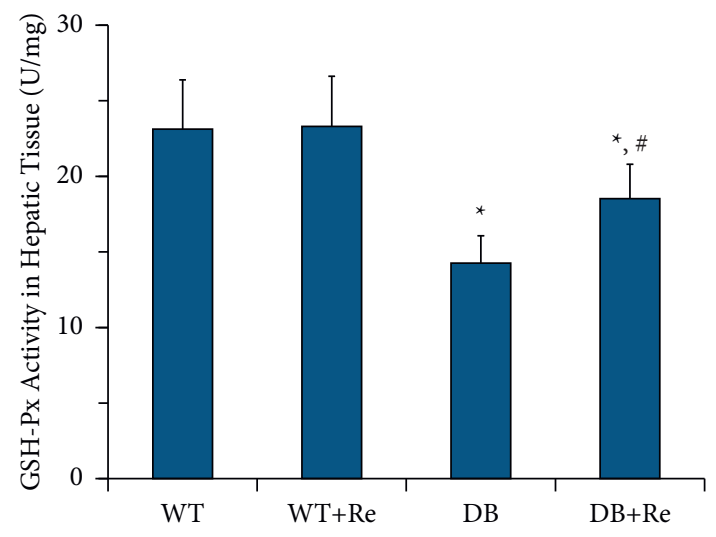

(d)

FIGURE 4: Re inhibits oxidative stress in hepatic tissue. (a) MDA levels in hepatic tissue; (b) activities of SOD in hepatic tissue; (c) activities of CAT in hepatic tissue; and (d) activities of GSH-Px in hepatic tissue. Data are mean \pm SD, and $n=8$. One-way ANOVA with Tukey's post hoc test was employed for group comparisons, with $P<0.05$ indicating statistical significance. ${ }^{*} P<0.05$ compared to the WT group; ${ }^{\#} P<0.05$ compared to the DB group. 


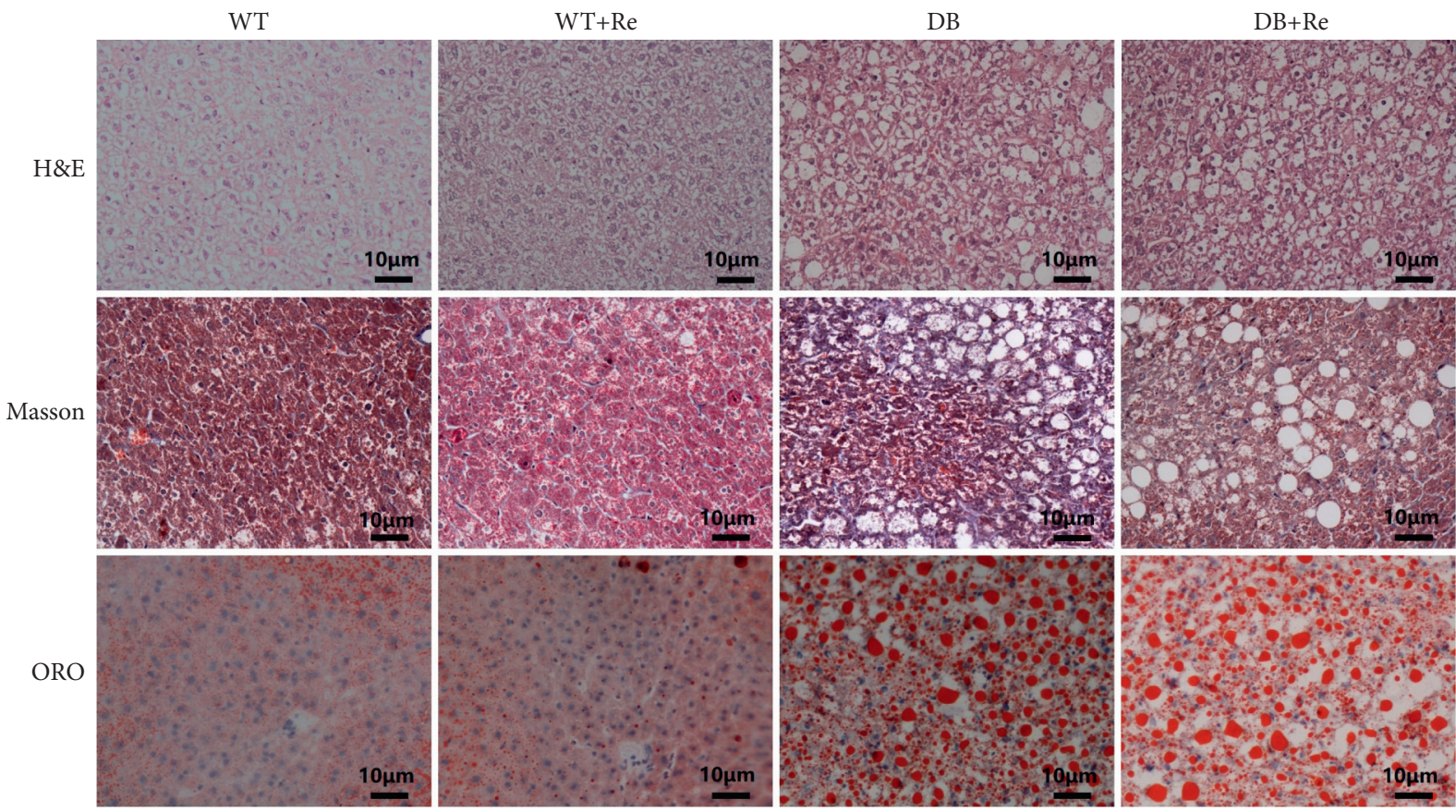

Figure 5: Re does not reverse simple steatosis but inhibits fibrosis in hepatic tissue. Representative H\&E, Masson, and ORO staining histology photomicrographs of the hepatic tissue in mice. All scale bars represent $10 \mu \mathrm{m}$.
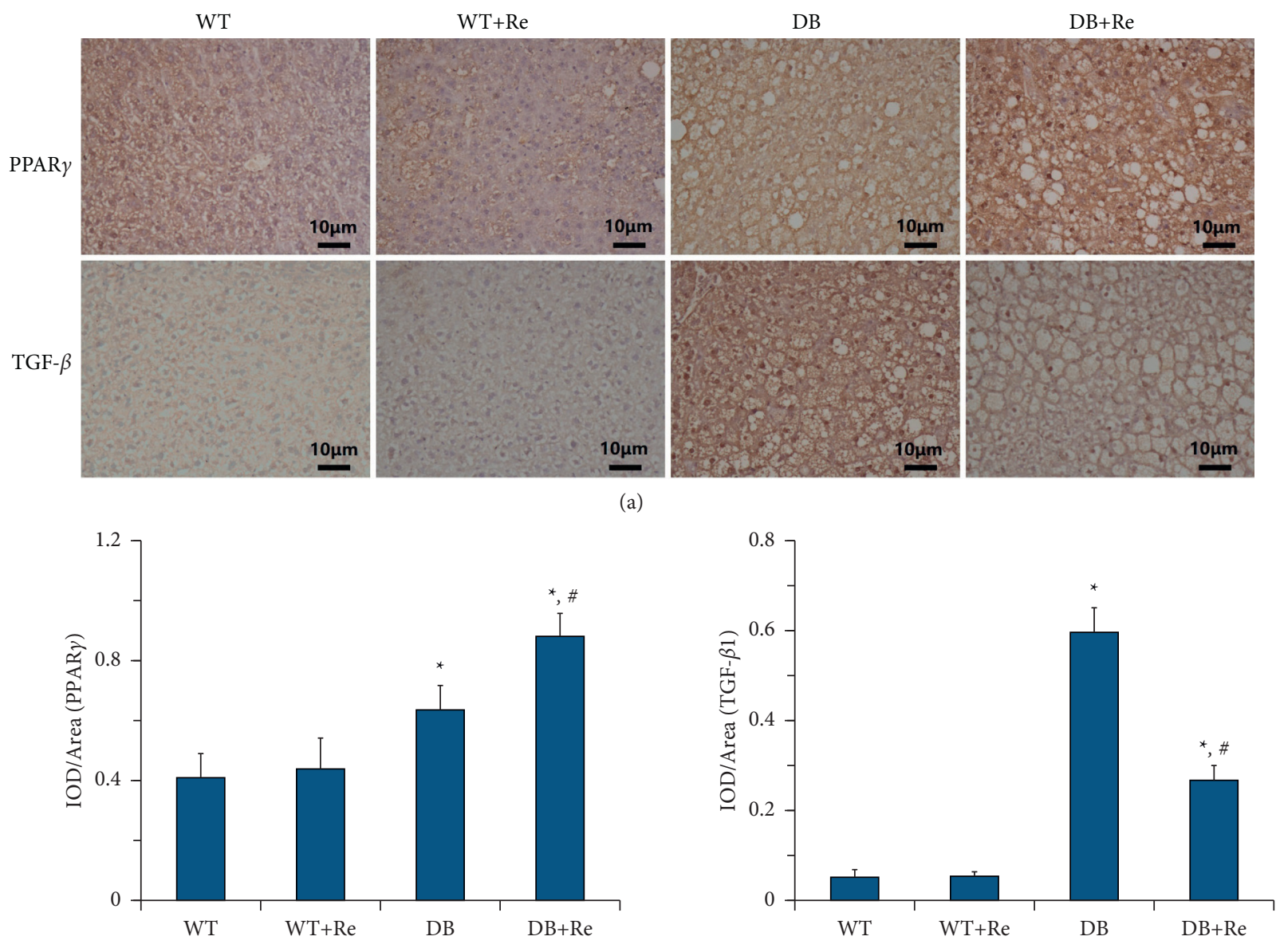

(b)

(c)

FIgURE 6: Re upregulates PPAR $\gamma$ and downregulates TGF- $\beta 1$ expression in hepatic tissue. (a) Representative IHC staining photomicrographs of the hepatic tissue in mice and quantitative results of IHC staining, which were presented as IOD/area and were proportional to the levels of PPAR $\gamma$ (b) and TGF- $\beta 1$ (c). All scale bars represent $10 \mu \mathrm{m}$. Data are mean \pm SD, and $n=4$. One-way ANOVA with Tukey's post hoc test was employed for group comparisons, with $P<0.05$ indicating statistical significance. ${ }^{*} P<0.05$ compared to the WT group; ${ }^{\#} P<0.05$ compared to the DB group. 


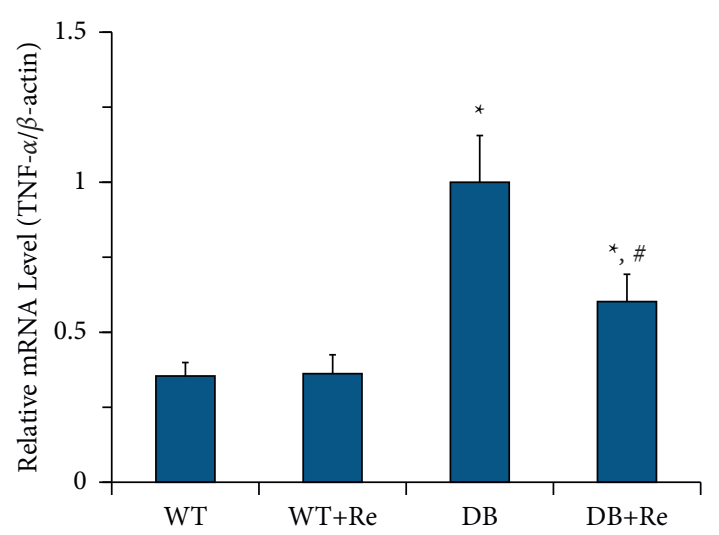

(a)

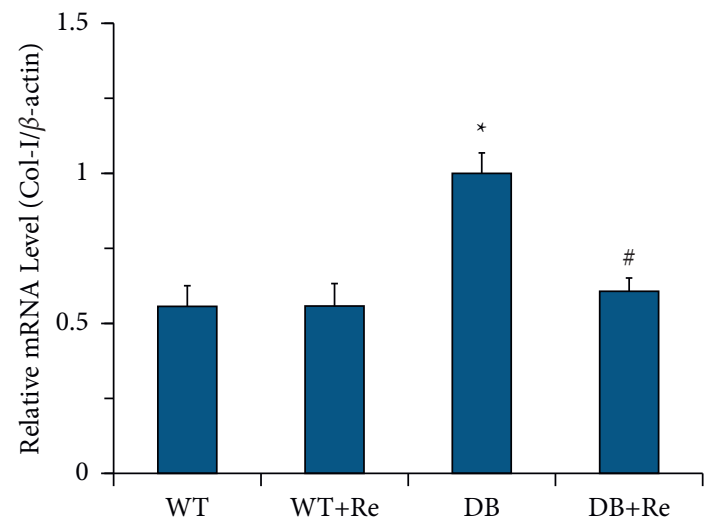

(c)

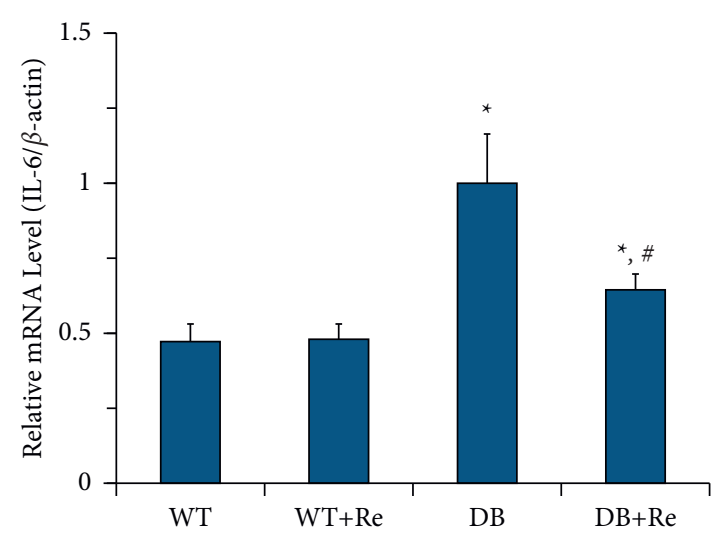

(b)

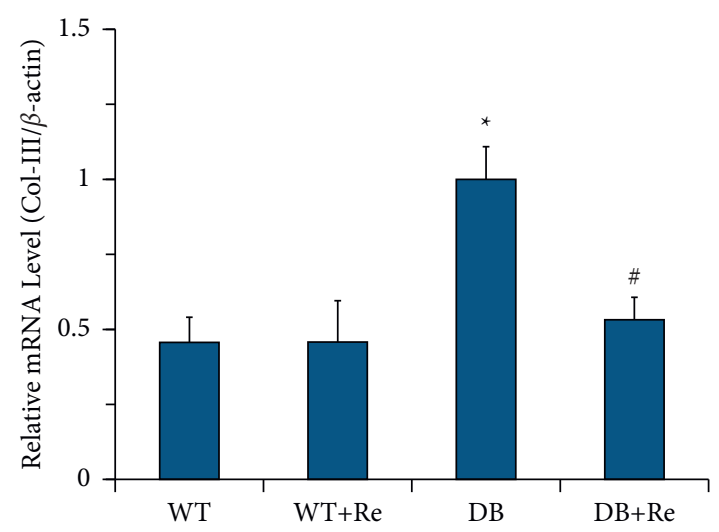

(d)

FIgURE 7: Re reduces levels of inflammatory cytokines and procollagens in hepatic tissue. Relative mRNA levels of TNF- $\alpha$ (a), IL-6 (b), Col-I (c), and Col-III (d). $\beta$-Actin was used as a housekeeping gene. Data are mean $\pm \mathrm{SD}$, and $n=4$. One-way ANOVA with Tukey's post hoc test was employed for group comparisons, with $P<0.05$ indicating statistical significance. ${ }^{*} P<0.05$ compared to the WT group; ${ }^{\#} P<0.05$ compared to the DB group.

Though NAFLD/NASH has a complex relationship with CHD and T2DM, few studies have been reported regarding using ZYC for NAFLD/NASH treatment. So, this study was performed to confirm that whether Re, an essential component of ZYC, could improve NAFLD in $\mathrm{db} / \mathrm{db}$ mice.

As the results in this study showed, although Re did not affect the body weight, blood glucose, and lipids of $\mathrm{db} / \mathrm{db}$ mice (Figure 2), it did control the progress of NAFLD, as the activities of aminotransferases in serum were reduced (Figure 3) and collagen deposition in hepatic tissue was attenuated (Figure 5).

As the first stage of NAFLD, simple steatosis in liver is inevitable in most T2DM patients, which also exist in $\mathrm{db} / \mathrm{db}$ mice. According to the "multihit hypothesis" of NAFLD [12-16], insulin resistance within the liver is implicated in the pathogenesis of NAFLD. TZDs, synthetic agonists of $\operatorname{PPAR} \gamma$, are the only current antidiabetic agents that increase insulin sensitivity as the main mechanism. Although TZDs have recently fallen into disuse in glycemic control due to concerns over side effects and adverse events, it is worthy to use them in T2DM patients with NAFLD $[19,27,28]$. In this study, Re upregulated the expression of PPAR $\gamma$ in hepatic tissue in $\mathrm{db} / \mathrm{db}$ mice (Figures 6(a) and 6(b)), and this might contribute to the effects of Re which improves inflammation and fibrosis in hepatic tissue (Figures 5 and 7). These effects were not able to reverse simple steatosis in hepatic tissue in $\mathrm{db} / \mathrm{db}$ mice, but did prevent it from developing into NASH or hepatic fibrosis (Figures 3 and 5).

In our previous studies $[6,7]$, we had demonstrated that Re improved CHD by reducing oxidative stress and TGF- $\beta$ levels, which are both important factors of the progress of NAFLD $[14,28,29]$. In this study, we demonstrated that Re reduced oxidative stress (Figure 4) and TGF- $\beta 1$ levels (Figures 6(a) and 6(c)), and these might also contribute to the anti-inflammation, antifibrosis, and hepatic protective effects of Re.

According to global research for decades, it is well recognized that ginsenosides, such as Re, improve a variety of cardiovascular and metabolic diseases, as well as their complication. The most common mechanisms were the antioxidation, anti-inflammation, and antifibrosis effects of ginsenosides, while the activation of PPAR $\gamma$ was also reported [30]. These were consistent with our findings in this study. 


\section{Conclusions}

Although no obvious effect on simple steatosis in the livers of $\mathrm{db} / \mathrm{db}$ mice was observed, Re indeed attenuates early hepatic injury, including mild ALT/AST elevation and collagen deposition. The mechanisms of improving inflammation and fibrosis in hepatic tissue might be associated to the upregulation of PPAR $\gamma$ expression and downregulation of oxidative stress. These indicated that ZYC, whose essential component is Re, might be able to help T2DM patients with NAFLD to control the progress of NAFLD as an alternation of TZDs, whose side effects and adverse events should not be ignored. Further studies and clinical trials are needed to be carried out before using in clinic.

\section{Data Availability}

The data used to support the findings of this study are included within the article.

\section{Ethical Approval}

All the experimental procedures involving animals were conducted in accordance with the Institutional Animal Care Guidelines of Jilin University and approved by the Laboratory Animal Ethics Committee of School of Pharmaceutical Sciences, Jilin University.

\section{Conflicts of Interest}

All the authors have no conflicts of interest to declare.

\section{Acknowledgments}

This study was supported by the National Natural Science Foundation of China (Grant 81473378) and Science and Technology Development Projects of Jilin Province (Grant 20170101002JC).

\section{References}

[1] Y. Qiao, J. Zhang, Y. Liu et al., "Efficacy and safety of zhenyuan capsule for coronary heart disease with abnormal glucose and lipid metabolism: study protocol for a randomized, double-blind, parallel-controlled, multicenter clinical trial," Evidence-based Complementary and Alternative Medicine, vol. 2018, Article ID 1716430, 2018.

[2] Y. Cao, W. Q. Wang, L. Lu, and X. M. Guo, "Adjuvant effects of Zhenyuan capsule on chronic heart failure: meta-analysis," Zhongguo Zhongyao Zazhi, vol. 42, no. 13, pp. 2583-2590, 2017.

[3] Y. Jiang, M. Li, Z. Lu et al., "Ginsenoside Rg3 induces ginsenoside Rb1-comparable cardioprotective effects independent of reducing blood pressure in spontaneously hypertensive rats," Experimental and therapeutic medicine, vol. 14, no. 5, pp. 4977-4985, 2017.

[4] H. Liu, Y. Jiang, M. Li, X. Yu, D. Sui, and L. Fu, "Ginsenoside $\mathrm{Rg} 3$ attenuates angiotensin II-mediated renal injury in rats and mice by upregulating angiotensin-converting enzyme 2 in the renal tissue," Evidence-based Complementary and Alternative Medicine, vol. 2019, Article ID 6741057, 2019.
[5] X. Liu, Y. Jiang, W. Fu, X. Yu, and D. Sui, "Combination of the ginsenosides $\mathrm{Rb} 3$ and $\mathrm{Rb} 2$ exerts protective effects against myocardial ischemia reperfusion injury in rats," International Journal of Molecular Medicine, vol. 45, no. 2, pp. 519-531, 2020.

[6] Q. W. Wang, X. F. Yu, H. L. Xu, Y. C. Jiang, X. Z. Zhao, and D. Y. Sui, "Ginsenoside Re attenuates isoproterenol-induced myocardial injury in rats," Evidence-based Complementary and Alternative Medicine, vol. 2018, Article ID 8637134, 2018.

[7] Q. W. Wang, X. F. Yu, H. L. Xu, X. Z. Zhao, and D. Y. Sui, "Ginsenoside Re improves isoproterenol-induced myocardial fibrosis and heart failure in rats," Evidence-based Complementary and Alternative Medicine, vol. 2019, Article ID 3714508, 2019.

[8] J. M. Hazlehurst, C. Woods, T. Marjot, J. F. Cobbold, and J. W. Tomlinson, "Non-alcoholic fatty liver disease and diabetes," Metabolism, vol. 65, no. 8, pp. 1096-1108, 2016.

[9] G. Targher, L. Bertolini, R. Padovani et al., "Prevalence of nonalcoholic fatty liver disease and its association with cardiovascular disease among type 2 diabetic patients," Diabetes Care, vol. 30, no. 5, pp. 1212-1218, 2007.

[10] R. M. Williamson, J. F. Price, S. Glancy et al., "Prevalence of and risk factors for hepatic steatosis and nonalcoholic Fatty liver disease in people with type 2 diabetes: the Edinburgh Type 2 Diabetes Study," Diabetes Care, vol. 34, no. 5, pp. 1139-1144, 2011.

[11] A. Lonardo, F. Nascimbeni, A. Mantovani, and G. Targher, "Hypertension, diabetes, atherosclerosis and NASH: cause or consequence?" Journal of Hepatology, vol. 68, no. 2, pp. 335-352, 2018.

[12] C. P. Day and O. F. W. James, "Steatohepatitis: a tale of two "hits"?" Gastroenterology, vol. 114, no. 4, pp. 842-845, 1998.

[13] C. L. Gentile and M. J. Pagliassotti, "The role of fatty acids in the development and progression of nonalcoholic fatty liver disease," The Journal of Nutritional Biochemistry, vol. 19, no. 9, pp. 567-576, 2008.

[14] J. K. Dowman, J. W. Tomlinson, and P. N. Newsome, "Pathogenesis of non-alcoholic fatty liver disease," QJM, vol. 103, no. 2, pp. 71-83, 2010.

[15] K. Cusi, "Role of obesity and lipotoxicity in the development of nonalcoholic steatohepatitis: pathophysiology and clinical implications," Gastroenterology, vol. 142, no. 4, pp. 711-725, 2012.

[16] W. Chang, H. Fa, D. Xiao, and J. Wang, "MicroRNA-184 alleviates insulin resistance in cardiac myocytes and high fat diet-induced cardiac dysfunction in mice through the LPP3/ DAG pathway," Molecular and Cellular Endocrinology, vol. 508, Article ID 110793, 2020.

[17] Y. Takahashi, Y. Soejima, A. Kumagai, M. Watanabe, H. Uozaki, and T. Fukusato, "Inhibitory effects of Japanese herbal medicines sho-saiko-to and juzen-taiho-to on nonalcoholic steatohepatitis in mice," PLoS One, vol. 9, no. 1, Article ID 87279, 2014.

[18] H. Kitade, G. Chen, Y. Ni, and T. Ota, "Nonalcoholic fatty liver disease and insulin resistance: new insights and potential new treatments," Nutrients, vol. 9, no. 4, Article ID 387, 2017.

[19] M. G. Radaelli, F. Martucci, S. Perra et al., "NAFLD/NASH in patients with type 2 diabetes and related treatment options," Journal of Endocrinological Investigation, vol. 41, no. 5, pp. 509-521, 2018.

[20] R. J. Henning, "Type-2 diabetes mellitus and cardiovascular disease," Future Cardiology, vol. 14, no. 6, pp. 491-509, 2018.

[21] C. C. Low Wang, C. N. Hess, W. R. Hiatt, and A. B. Goldfine, "Clinical update: cardiovascular disease in diabetes mellitus: 
atherosclerotic cardiovascular disease and heart failure in type 2 diabetes mellitus - mechanisms, management, and clinical considerations," Circulation, vol. 133, no. 24, pp. 2459-2502, 2016.

[22] D. P. Kumar, R. Caffrey, J. Marioneaux et al., "The PPAR $\alpha / \gamma$ agonist saroglitazar improves insulin resistance and steatohepatitis in a diet induced animal model of nonalcoholic fatty liver disease," Scientific Reports, vol. 10, no. 1, Article ID 9330, 2020.

[23] J. N. Van der Veen, S. Lingrell, X. Gao et al., "Pioglitazone attenuates hepatic inflammation and fibrosis in phosphatidylethanolamine N-methyltransferase-deficient mice," American Journal of Physiology - Gastrointestinal and Liver Physiology, vol. 310, no. 7, pp. 526-538, 2016.

[24] Q. M. Anstee and R. D. Goldin, "Mouse models in non-alcoholic fatty liver disease and steatohepatitis research," International Journal of Experimental Pathology, vol. 87, no. 1, pp. 1-16, 2006.

[25] Q. Wang, L. Jiang, J. Wang et al., “Abrogation of hepatic ATPcitrate lyase protects against fatty liver and ameliorates hyperglycemia in leptin receptor-deficient mice," Hepatology, vol. 49, no. 4, pp. 1166-1175, 2009.

[26] K. J. Livak and T. D. Schmittgen, "Analysis of relative gene expression data using real-time quantitative PCR and the 2(Delta Delta C(T)) Method," Methods, vol. 25, no. 4, pp. 402-408, 2001.

[27] R. E. Soccio, E. R. Chen, and M. A. Lazar, "Thiazolidinediones and the promise of insulin sensitization in type 2 diabetes," Cell Metabolism, vol. 20, no. 4, pp. 573-591, 2014.

[28] C. Peng, A. G. Stewart, O. L. Woodman, R. H. Ritchie, and C. X. Qin, "Non-Alcoholic steatohepatitis: a review of its mechanism, models and medical treatments," Frontiers in Pharmacology, vol. 11, Article ID 603926, 2020.

[29] K. Pafili and M. Roden, "Nonalcoholic fatty liver disease (NAFLD) from pathogenesis to treatment concepts in humans," Molecular Metabolism, vol. 50, Article ID 101122, 2020.

[30] L. Bai, J. Gao, F. Wei, J. Zhao, D. Wang, and J. Wei, "Therapeutic potential of ginsenosides as an adjuvant treatment for diabetes," Frontiers in Pharmacology, vol. 9, Article ID 423, 2018. 